\title{
Termination of pregnancy at very early gestation without visible yolk sac on ultrasound
}

\author{
Rebecca Heller, ${ }^{1}$ Sharon Cameron ${ }^{2}$
}

\begin{abstract}
${ }^{1}$ Clinical Research Fellow, Chalmers Sexual \& Reproductive Health Service, Edinburgh, UK ${ }^{2}$ Consultant Gynaecologist, Chalmers Sexual \& Reproductive Health Service, Edinburgh, UK

\section{Correspondence to Dr Rebecca Heller, Chalmers Sexual \& Reproductive Health Service, 2A Chalmers Street, Edinburgh EH3 9ES, UK; rheller@staffmail.ed.ac.uk}

Received 4 March 2014 Revised 3 July 2014 Accepted 25 July 2014 Published Online First 8 September 2014
To cite: Heller $\mathrm{R}$, Cameron $\mathrm{S}$. J Fam Plann Reprod Health Care 2015;41:90-95.

\begin{abstract}
Introduction Requests for termination of pregnancy (TOP) at very early gestation ( $\leq 6$ weeks) can prove challenging for abortion services as the ultrasound feature usually accepted as definitive evidence of an intrauterine pregnancy (IUP), the presence of a yolk sac within a gestational sac, may not yet be evident. In 2011 the Edinburgh TOP service introduced a protocol permitting women to proceed to treatment without further investigations provided that ultrasound showed the features of an eccentrically placed gestational sac ( $\geq 3 \mathrm{~mm}$ ) with a decidual reaction, and there were no signs, symptoms or risk factors for ectopic pregnancy. Methods A retrospective audit was conducted

\section{Key message points}

- Women whose ultrasound scan shows an eccentrically placed gestational sac with decidual reaction, without risk factors for ectopic pregnancy, can proceed to termination of pregnancy (TOP) without further investigations.

- Adopting this practice would reduce the number of visits women need to make to clinics, minimising distress and reducing service workload.

- Medical TOP at very early gestation when no yolk sac is visible appears to have high efficacy.
\end{abstract} of outcomes of women presenting for TOP at $\leq 6$ weeks' gestation over a 2-year period using the hospital computerised database.

Results A total of 1155 women presented for TOP with an ultrasound gestational age of $\leq 6$ weeks. Of these, $1030(89 \%)$ had ultrasound evidence of a yolk sac. Eighty-seven women (7.5\%) had an eccentrically placed gestational sac with a decidual reaction. All 87 women fulfilled our criteria to proceed to medical TOP, and 66 did so. In the remaining 21 cases, further investigations were performed before they proceeded to medical TOP. Two $(0.17 \%)$ medical TOPs failed, both in women whose initial ultrasound had shown a yolk sac.

Conclusion Women with ultrasound features consistent with a very early IUP $(\geq 3 \mathrm{~mm}$ eccentrically placed gestational sac with a decidual reaction) and without signs, symptoms or risk factors for ectopic pregnancy can proceed directly to medical TOP without the need for delay for further ultrasonography.

\section{CrossMark}

\section{INTRODUCTION}

The widespread availability of highly sensitive urine pregnancy tests means that women with an unintended pregnancy may present very soon after a missed period to request a termination of pregnancy (TOP). Although there is a paucity of data on abortions under 6 weeks, evidence suggests that the earlier a TOP is performed, the safer it is. ${ }^{1}$ National guidelines also emphasise the importance of minimising unnecessary delays in referring women who request TOP. $^{2}$ The Royal College of Obstetricians \& Gynaecologists (RCOG) recommends that women requesting TOP should be referred for assessment within five working days of the request. ${ }^{2}$ This recommendation is of particular importance since women in Great Britain requesting a TOP often choose the early medical method. $^{3}$

Ultrasound is widely recognised as the age. ${ }^{2}{ }^{4}$ However, for women at very early gestations ( $<6$ weeks' amenorrhoea), the ultrasound feature that is traditionally accepted as definitive evidence of an intrauterine pregnancy (IUP), namely the presence of a yolk sac within a gestational gold standard for assessing gestational 
sac, may not yet be visible. ${ }^{5-9}$ In addition, the vigilance of clinicians in trying to exclude potential ectopic pregnancies can lead to women who present for TOP before 6 weeks' gestation having their treatment delayed, due to a perceived need to visualise a yolk sac on ultrasound. Women may also be subjected to unnecessary blood tests for serial measurement of human chorionic gonadotrophin (hCG) to confirm that the pregnancy is intrauterine. This causes inconvenience for women and may add to the distress of an unintended pregnancy. More appointments may add to the workload of clinics, making it more difficult for new referrals to be seen promptly.

The Royal Infirmary of Edinburgh (RIE) is the main provider of TOP services for Lothian, Scotland, UK (Edinburgh and surrounding region). ${ }^{10}$ All women requesting a TOP at this service who fulfil the terms of the 1967 Abortion Act ${ }^{11}$ have a transabdominal ultrasound scan performed by an ultrasonographer to assess gestational age. A transvaginal scan is performed if suboptimal views are obtained. Prior to 2011, all patients with no visible yolk sac were brought back 1 week later for a repeat ultrasound scan. However, with the need to minimise the wait between referral for TOP and assessment in line with RCOG recommendations, ${ }^{2}$ clinicians were reporting that increasing numbers of women were presenting for assessment at earlier gestations without ultrasound evidence of a yolk sac. This resulted in further clinic appointments for repeat ultrasound scans, and delay in treatment.

In January 2011 the ultrasound criteria for treatment were reviewed against current evidence for early IUP and a new protocol was introduced. This protocol allowed women with less than 6 weeks' amenorrhoea, without symptoms of ectopic pregnancy (pain or bleeding), and without risk factors for ectopic pregnancy (history of sterilisation, tubal surgery or previous ectopic pregnancy), to proceed directly to a medical TOP without the need for further scans or serum hCG measurements if the criteria in Box 1 were met. The ultrasound criteria were an eccentrically placed gestation sac of $\geq 3 \mathrm{~mm}$ within a markedly thickened decidua (Figure 1). These ultrasound criteria have been shown to be consistent with a very early IUP. ${ }^{9}$ Women who fulfilled all the protocol criteria were treated exactly as women in whom a yolk sac was visualised. For women not fulfilling these criteria, further clinic visits for serial hCGs and ultrasound scans to exclude ectopic pregnancy were necessary. Women with suspected or confirmed ectopic pregnancy were referred to the hospital-based Early Pregnancy Unit.

The aims of this study were to determine adherence to the protocol, and to evaluate what proportion of women who presented at an early gestation, who would formerly have had to delay treatment until ultrasound evidence of a yolk sac was present, were
Box 1 Criteria to be met for women without yolk sac or fetal pole visible on ultrasound scan to proceed to termination of pregnancy

Ultrasound

- Intrauterine gestation sac 3-20 mm

- Eccentrically placed

- Decidual reaction visible

Clinical

- No symptoms suggestive of ectopic pregnancy (pain, bleeding)

- No significant risk factors for ectopic pregnancy (sterilisation, tubal surgery, previous ectopic pregnancy)

- Last menstrual period consistent with a pregnancy of less than 6 weeks' gestation.

able to be treated without the need for further visits or investigations.

\section{METHODS}

A retrospective case note review was conducted of all women attending the RIE TOP clinic during the 2-year period January 2011 to December 2012, whose ultrasound scan identified them as being at 6 weeks or less gestation at their first visit and who proceeded with a TOP. Women who decided to continue with their pregnancy were not included. Cases were then subdivided into those whose scan showed (1) a yolk sac or fetal pole, (2) an intrauterine sac only or (3) an empty uterus. For those cases with an intrauterine sac only, the scan reports were scrutinised to determine if comments had been made on its size, whether its position in the uterine cavity was eccentric, and whether a decidual reaction was present. For all cases of 6 weeks or less gestation the regional hospital computerised record system (TRAK) was searched to determine the number of visits made to the clinic, including the number of scans performed, the number of serum hCG tests taken and the

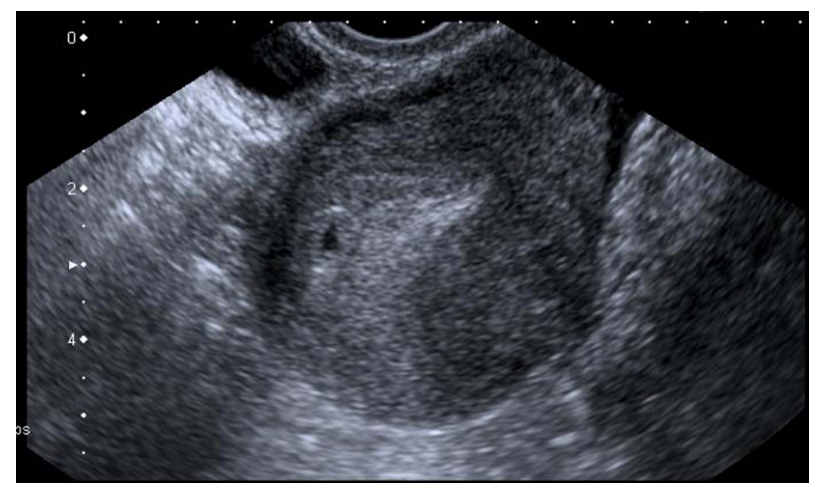

Figure 1 Eccentrically placed gestational sac with decidual reaction. 
eventual outcome of the pregnancy. The maternity database was checked to exclude the possibility that a woman may have had a failed TOP that proceeded to delivery. The quality improvement team for gynaecology at RIE approved the project. The chair of the local ethics committee also reviewed the project proposal and confirmed that ethical approval was not required.

\section{RESULTS}

A total of 1155 women presented for a TOP with an ultrasound scan suggestive of a pregnancy of less than 6 weeks' gestation during the 2-year period (Figure 2).

In $1030(89 \%)$ of these cases there was ultrasonic evidence of either a fetal pole or either a yolk sac and all of these women proceeded to TOP. Thirteen $(1.5 \%)$ women with a yolk sac present on initial assessment had a surgical TOP (vacuum aspiration under general anaesthesia). The remainder had a medical TOP (200 mg oral mifepristone followed 2436 hours later by $800 \mu \mathrm{g}$ misoprostol vaginally, with the option to remain in the hospital or to pass the products of conception at home). Two (0.19\%) women were found to have ongoing pregnancies after a failed medical TOP. In both women, a fetal pole was present on ultrasound at initial presentation. Both went on to have a successful repeat medical TOP.

Eighty-seven $(7.5 \%)$ women with gestations of $\leq 6$ weeks had an eccentrically placed sac of $\geq 3 \mathrm{~mm}$ with a decidual reaction noted on ultrasound scan, meeting the study's protocol criteria. Of these, 66 (75.9\%) proceeded direct to TOP as per protocol recommendations. Of the remaining women, 12 $(13.7 \%)$ had one or more serum hCGs before TOP, six $(6.8 \%)$ had a further ultrasound 1 week after initial presentation, and three $(3.4 \%)$ had both $\geq 1$ serum hCGs and a second ultrasound scan. In all of these cases repeat ultrasound and/or serum hCGs confirmed an IUP and these women proceeded to a medical TOP (Figure 2). There were no ectopic pregnancies or medical TOP failures in this group of women.

Seventeen women had ultrasound reports either of an eccentrically placed intrauterine sac but without a decidual reaction, or of a sac with a decidual reaction but that was centrally placed. Sixteen women had an empty uterus at their first attendance at clinic, despite a positive pregnancy test. One of these women had an

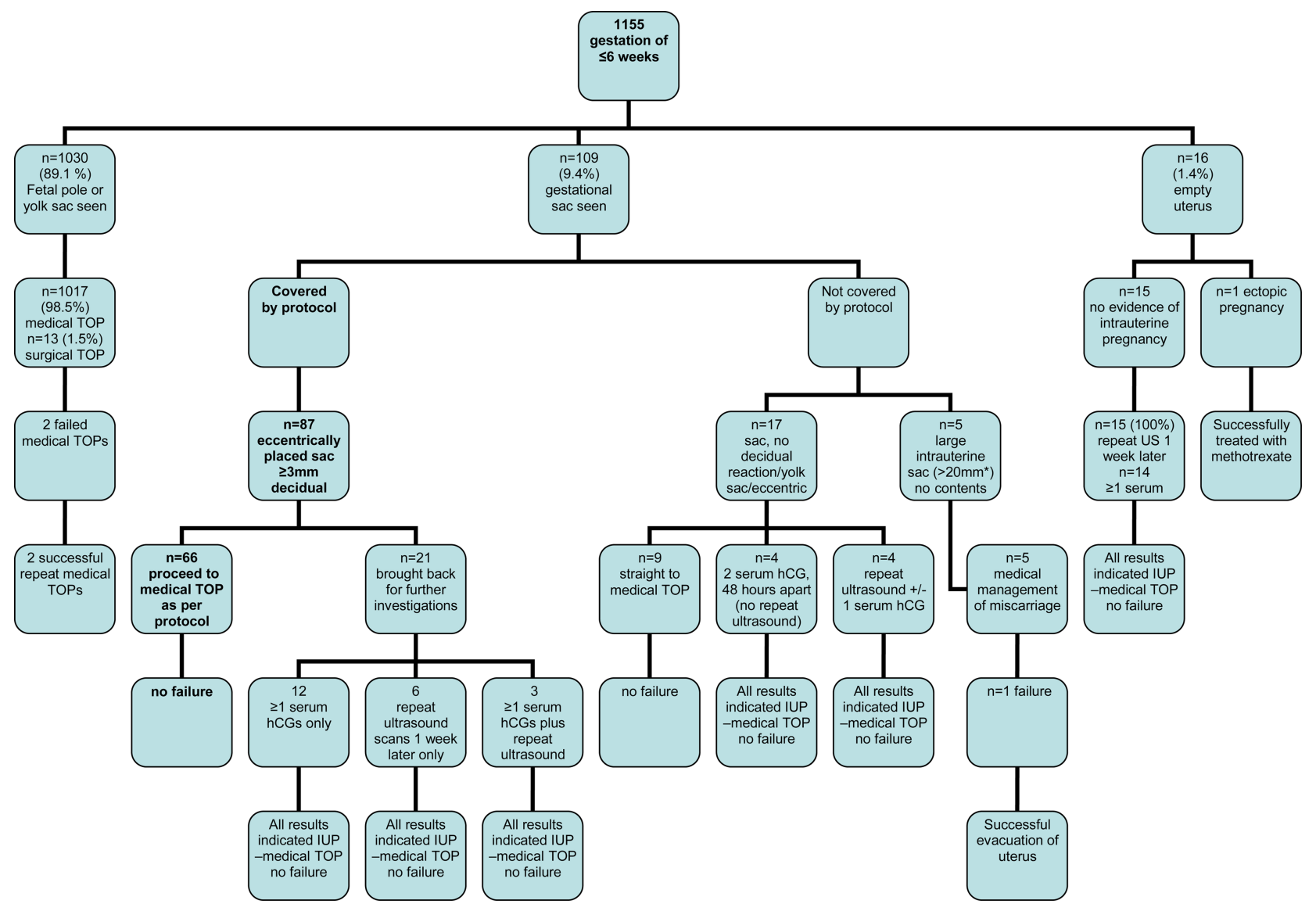

Figure 2 Ultrasound features and outcomes for 1155 women requesting termination of pregnancy at $\leq 6$ weeks' gestation. hCG, human chorionic gonadotrophin; IUP, intrauterine pregnancy; TOP, termination of pregnancy; US, ultrasound. ${ }^{*}>25 \mathrm{~mm}$ after changes to guidelines in November $2011 .^{12}$ 
adnexal mass on ultrasound at presentation and her ectopic pregnancy was managed with methotrexate by the Early Pregnancy Unit at the RIE. The remaining 5 women were managed as shown in Figure 2.

Thus, out of 1155 women with ultrasound scans suggesting a gestation of $\leq 6$ weeks, only $2.8 \%(n=32)$ had findings necessitating a repeat ultrasound and/or serum hCG according to the protocol.

\section{DISCUSSION}

Our study demonstrated that even among presentations to a TOP service at less than 6 weeks' gestation it is usually possible to visualise a yolk sac on ultrasound scanning, which is generally accepted as being indicative of an IUP. For most of the remaining women the use of our ultrasound and clinical criteria (eccentrically placed gestation sac, decidual reaction, no risk factors or signs or symptoms of ectopic pregnancy) to indicate very early IUP permits the majority of the remainder to proceed to an early medical TOP.

Clinicians who are concerned that guidelines mandating reduced waiting times for referral for TOP assessment could result in a greater proportion of women presenting before an IUP can be confirmed may be reassured by our findings. Even if we had insisted upon visualisation of a yolk sac on ultrasound, only $125 / 1155(10.8 \%)$ women with gestations of $\leq 6$ weeks had no visible yolk sac and would therefore have required a further visit. By adopting the ultrasound and clinical criteria for very early IUP used here, our study suggests that this could be reduced to only $2.8 \%$ requiring an extra visit. It is also reassuring that the medical TOP failure rate was no higher in this very early group than in women with ultrasound evidence of a yolk sac or fetal pole.

One asymptomatic ectopic pregnancy was found. In a review of ectopic pregnancy the authors concluded that $97 \%$ of women with an ectopic pregnancy have a history or a presenting complaint of abdominal pain, $79 \%$ a history or presentation of vaginal bleeding and $91 \%$ a history or presentation of abdominal tenderness. ${ }^{13}$ Our protocol makes it clear that women with clinical signs or symptoms should undergo further investigations. It remains important that women who do not fulfil our criteria for IUP are followed up rigorously. There is evidence that the frequency of ectopic pregnancies amongst women following TOP treatment may be lower than in the general population, with one review of 57 studies of 46421 women who had undergone TOP with various regimens reporting only 10 ectopics. ${ }^{14}$

In order to meet our protocol an eccentrically placed sac with a decidual reaction must be seen and documented. This has been described as a sac outlined by an echogenic rim due to the trophoblast invasion into the surrounding decidual endometrium. ${ }^{8}{ }^{9}$ The use of a 'decidual reaction' to infer the presence of an IUP has provoked some controversy, and there is evidence that this feature cannot always be seen. ${ }^{15} 16$ The presence of a decidual reaction is not always apparent in pregnancies that eventually prove to be intrauterine. $^{8} 9$ This is why it comprises just one of the elements of a protocol that consists of both ultrasound and clinical features. However, results can be improved by ultrasonographers looking intentionally for the uterine cavity line at the time of ultrasound scanning, rather than studying still images afterwards. ${ }^{17}$ The main concern in the literature is that if no decidual reaction is seen, an ectopic pregnancy will be assumed and that treatment such as methotrexate will be given in a potentially viable IUP. ${ }^{16}$

If a sac without eccentric placement or decidual reaction is seen, a pseudosac in the presence of an ectopic pregnancy must be excluded. In our service, women with a centrally placed sac or with no decidual reaction seen underwent further investigations to exclude ectopic pregnancy. Evidence suggests that pseudosacs are uncommon, and are present in fewer than $10 \%$ of ectopic pregnancies. ${ }^{15} 161819$ Although the possibility of ectopic pregnancy is one that must not be overlooked, the commonest outcome associated with a gestational sac without any decidual reaction would be a failing or continuing IUP. ${ }^{6}$

In a small minority of our patients in whom a gestational sac was seen, no comment on the presence of a decidual reaction was documented by the ultrasonographer. Unfortunately, as this was a retrospective audit, we do not know the explanation for these omissions. It is possible that some ultrasonographers were not aware of the protocol or the importance of documenting this information. Neither can we exclude the possibility that the presence or absence of a decidual reaction may have been confirmed verbally with the ultrasonographer in cases where women proceeded to a medical TOP without further investigation.

There is limited literature on the consequences of proceeding with medical TOP for pregnancies of 6 weeks or less. The challenge may be a relatively new one; a consequence of home pregnancy tests being so sensitive $^{2021}$ and the routine use of, and increased reliance upon, ultrasound in TOP services, at least in high-income settings. ${ }^{2} 4$

Most literature concerning the use of ultrasound in medical TOP examines the necessity of using ultrasound at all, and therefore points out the improved dating accuracy, as well as the benefits of improved diagnosis of ectopic pregnancies and visualisation of uterine cavity abnormalities or ovarian pathology. ${ }^{4}$ 22-26 However, other studies question the need for ultrasound, given the good correlation between gestational age using last menstrual period and clinical assessment. ${ }^{23} 2728$

A small number of studies have reported the outcome of medical TOP in women who have no visible gestational sac on ultrasound. ${ }^{29-31}$ Some investigators suggest that the failure rate of medical TOP may 
be higher in these very early gestations (ongoing pregnancy rates of $7.0-7.5 \%){ }^{29} 30$ The explanation for this is unclear, but could be because some very early cases might include women with early miscarriages. There is good evidence that medical methods are less effective for management of miscarriage than for terminating a viable pregnancy, with reported failure rates between $13 \%$ and $19 \% .^{32}$ It has also been postulated that very early pregnancies might be less sensitive to the effects of mifepristone and prostaglandins, since one small study of 'menstrual regulation' where a combination of mifepristone taken the day before the expected day of menstruation, followed by oral misoprostol 48 hours later, was associated with an overall continuing pregnancy (failure) rate of $4 \% .^{33}$

To our knowledge ours is the first study to examine outcomes of medical TOP at less than 6 weeks' gestation in women with ultrasound features of an intrauterine gestational sac only. Clearly our study is limited by the fact that the overall number of women who fulfilled our criteria to proceed directly to medical TOP was small. Furthermore, all ultrasound scans were performed by experienced ultrasonographers who specialise in gynaecological scanning, so our results may not be applicable to services with lower levels of ultrasound expertise. It is worth noting, however, that RCOG guidelines state that the use of ultrasound is not a requirement for a TOP service. ${ }^{2}$

\section{CONCLUSIONS}

Our study provides evidence that performing medical TOP for pregnancies of very early gestation, before ultrasound evidence of a yolk sac, is effective and appropriate, provided that there are no symptoms or risk factors suggestive of an ectopic pregnancy. Doing so avoids further clinic visits with concomitant delays in treatment for women.

Further larger studies are now required to confirm our findings. Other TOP services that rely heavily on ultrasound for assessment of gestational age could consider adopting this protocol.

Acknowledgements The authors wish to thank Anne Day, ultrasonographer, for providing the ultrasound image, and acknowledge Dr W C Duncan, Senior Lecturer in Reproductive Medicine, and Dr C West, Consultant Gynaecologist, for instigating the protocol originally.

Competing interests None.

Provenance and peer review Not commissioned; externally peer reviewed.

\section{REFERENCES}

1 World Health Organization (WHO). Safe Abortion: Technical and Policy Guidance for Health Care Systems (2nd edn). 2012. http://www.who.int/reproductivehealth/publications/unsafe abortion/9789241548434/en/ [accessed 17 October 2013].

2 Royal College of Obstetricians and Gynaecologists. The Care of Women Requesting Induced Abortion (Guideline No. 7). 2011.
http://www.rcog.org.uk/files/rcog-corp/Abortion\%20guideline_ web_1.pdf [accessed 17 October 2013].

3 Ingham R, Lee E. Evaluation of Early Medical Abortion (EMA) Pilot Sites. London, UK: Department of Health, 2008.

$4 \mathrm{McGalliard}$ C, Gaudoin M. Routine ultrasound for pregnancy termination requests increases women's choice and reduces inappropriate treatments. BJOG 2004;111:79-82.

5 Bottomley C, Van Belle V, Mukri F, et al. The optimal timing of an ultrasound scan to assess the location and viability of an early pregnancy. Hum Reprod 2009;24:1811-1817.

6 Bottomley C, Van Belle V, Pexsters A, et al. A model and scoring system to predict outcome of intrauterine pregnancies of uncertain viability. Ultrasound Obstet Gynecol 2011;37:588-595.

7 Elson J, Salim R, Tailor A, et al. Prediction of early pregnancy viability in the absence of an ultrasonically detectable embryo. Ultrasound Obstet Gynecol 2003;21:57-61.

8 Jurkovic D, Gruboeck K, Campbell S. Ultrasound features of normal early pregnancy development. Curr Opin Obstet Gynecol 1995;7:493-504.

9 Cacciatore B, Tiitinen A, Stenman U, et al. Normal early pregnancy: serum hCG levels and vaginal ultrasonography findings. BJOG 1990;97:899-903.

10 Information Services Division. Abortion Statistics 2012. May 2013. http://www.isdscotland.org/Health-Topics/Sexual-Health/ Publications/2013-05-28/2013-05-28-Abortions-Report.pdf? 59875124693 [accessed 17 October 2013].

11 The Abortion Act 1967. London, UK: HMSO, 1967.

12 Royal College of Obstetricians \& Gynaecologists. The Management of Early Pregnancy Loss (Addendum to Green Top Guideline No. 25 October 2006). October 2011. http:// www.rcog.org.uk/files/rcog-corp/Addendum\%20to\%20GTG\% 20No\%2025.pdf [accessed 20 January 2014].

13 Tay JI, Moore J, Walker JJ. Ectopic pregnancy. BMJ 2000;320:916-919.

14 Shannon C, Brothers LP, Philip NM, et al. Ectopic pregnancy and medical abortion. Obstet Gynecol 2004;104:161-167.

15 Doubilet PM, Benson CB. Double sac sign and intradecidual sign in early pregnancy: interobserver reliability and frequency of occurrence. J Ultrasound Med 2013;32:1207-1214.

16 Doubilet PM, Benson CB. First, do no harm ... to early pregnancies. J Ultrasound Med 2010;29:685-689.

17 Yeh $\mathrm{H}$. Use of the double decidual sac sign and intradecidual sign. J Ultrasound Med 2011;30:119-120.

18 Fleischer A, Pennell R, McKee M, et al. Ectopic pregnancy: features at transvaginal sonography. Radiology 1990;174:375-378.

19 Hill L, Kislak S, Martin J. Transvaginal sonographic detection of the pseudogestational sac associated with ectopic pregnancy. Obstet Gynecol 1990;75:986-988.

20 Tomlinson C, Marshall J, Ellis J. Comparison of accuracy and certainty of results of six home pregnancy tests available over-the-counter. Curr Med Res Opin 2008;24: $1645-1649$.

21 Johnson S, Shaw R, Parkinson P, et al. Home pregnancy test compared to standard-of-care ultrasound dating in the assessment of pregnancy duration. Curr Med Res Opin 2011;27:393-401.

22 Edwards J, Carson S. New technologies permit safe abortion at less than six weeks' gestation and provide timely detection of ectopic gestation. Am J Obstet Gynecol 1997;176:1101-1106.

23 Kulier R, Kapp N. Comprehensive analysis of the use of pre-procedure ultrasound for first- and second-trimester abortion. Contraception 2011;83:30-33. 
24 Fielding SL, Schaff EA, Nam N. Clinicians' perception of sonogram indication for mifepristone abortion up to 63 days. Contraception 2002;66:27-31.

25 Demianczuk N, Ven Den Hof M, Farquahrson D, et al. The use of first trimester ultrasound. J Obstet Gynecol Can 2003;25:864-875.

26 Blanchard K, Cooper D, Dickson K, et al. A comparison of women's, providers' and ultrasound assessment of pregnancy duration among termination of pregnancy clients in South Africa. BJOG 2007;114:569-575.

27 Bracken H, Clark W, Lichtenberg ES, et al. Alternatives to routine ultrasound for eligibility assessment prior to early termination of pregnancy with mifepristone-misoprostol. BJOG 2011;118:17-23.

28 Ellertson C, Elub B, Ambardekar S, et al. Accuracy of assessment of pregnancy duration by women seeking early abortions. Lancet 2000;355:877-881.
29 Schaff EA, Fielding SL, Eisinger S, et al. Mifepristone and misoprostol for early abortion when no gestational sac is present. Contraception 2001;63:251-254.

30 Goldstone P, Michelson J, Williamson E. Effectiveness of early medical abortion using low-dose mifepristone and buccal misoprostol in women with no defined intrauterine gestational sac. Contraception 2013;87:855-858.

31 Wiebe ER. Methotrexate with or without misoprostol to terminate pregnancies with no gestational sac visible by ultrasound. Int J Gynaecol Obstet 2009;107:64-65.

32 Zhang J, Gilles JM, Barnhart K, et al. A comparison of medical management with misoprostol and surgical management for early pregnancy failure. N Engl J Med 2005;353:761-769.

33 Swahn M, Bygdeman M, Jun-kang C, et al. Once-a-month treatment with a combination of mifepristone and the prostaglandin analogue misoprostol. Hum Reprod 1999;14:485-488. 